\title{
WATER AT POSITIVE AND NEGATIVE PRESSURES
}

\author{
H. E. STANLEY,${ }^{1}$ M. C. BARBOSA, ${ }^{1,2}$ S. MOSSA, ${ }^{1}$ P. A. NETZ, ${ }^{3}$ \\ F. SCIORTINO ${ }^{4}$ F. W. STARR,${ }^{5}$ and M. YAMADA ${ }^{1}$ \\ ${ }^{1}$ Center for Polymer Studies and Department of Physics, Boston University \\ Boston, MA 02215 USA \\ ${ }^{2}$ Instituto de Física, Universidade Federal do Rio Grande do Sul, Caixa Postal 15051 \\ 91501-970, Porto Alegre, RS, Brazil \\ ${ }^{3}$ Departamento de Química, Universidade Luterana do Brasil \\ 92420-280, Canoas, RS, Brazil \\ ${ }^{4}$ Dipartimento di Fisica Università di Roma La Sapienza, Istituto Nazionale di Fisica \\ della Materia, and INFM Center for Statistical Mechanics and Complexity, \\ Piazzale Aldo Moro 2, 00185 Roma, Italy \\ ${ }^{5}$ Polymers Division and Center for Theoretical and Computational Materials Science \\ National Institute of Standards and Technology, Gaithersburg, MD 20899 USA
}

\begin{abstract}
We review recent results of molecular dynamics simulations of two models of liquid water, the extended simple point charge (SPC/E) and the Mahoney-Jorgensen transferable intermolecular potential with five points (TIP5P), which is closer to real water than previously-proposed classical pairwise additive potentials. Simulations of the TIP5P model for a wide range of deeply supercooled states, including both positive and negative pressures, reveal (i) the existence of a non-monotonic "nose-shaped" temperature of maximum density (TMD) line and a nonreentrant spinodal, (ii) the presence of a low temperature phase transition. The TMD that changes slope from negative to positive as $\mathrm{P}$ decreases and, notably, the point of crossover between the two behaviors is located at ambient pressure (temperature $\approx 4 \mathrm{C}$, and density $\approx 1 \mathrm{~g} / \mathrm{cm}^{3}$ ). Simulations on the dynamics of the SPC/E model reveal (iii) the dynamics at negative pressure shows a minimum in the diffusion constant $D$ when the density is decreased at constant temperature, complementary to the known maximum of $D$ at higher pressures, and (iv) the loci of minima of $D$ relative to the spinodal shows that they are inside the thermodynamically metastable regions of the phase-diagram. These dynamical results reflect the initial enhancement and subsequent breakdown of the tetrahedral structure and of the hydrogen bond network as the density decreases.
\end{abstract}




\section{Introduction}

Water is an important liquid in nature, and is also fundamental in chemical and technological applications. Although the individual water molecule has a simple chemical structure, water is considered a complex fluid because of its anomalous behavior $[1,2,3,4,5,6]$. It expands on freezing and, at a pressure of $1 \mathrm{~atm}$, the density has a maximum at $4^{\circ} \mathrm{C}$. Additionally, there is a minimum of the isothermal compressibility at $46^{\circ} \mathrm{C}$ and a minimum of the isobaric heat capacity at $35^{\circ} \mathrm{C}$ [7]. These anomalies are linked with the microscopic structure of liquid water, which can be regarded as a transient gel — a highly associated liquid with strongly directional hydrogen bonds $[8,9]$. Each water molecule acts as both a donor and an acceptor of bonds, generating a structure that is locally ordered, similar to that of ice, but maintaining the longrange disorder typical of liquids. Despite the extensive work that has been done on water, many aspects of its behavior remain unexplained.

Several scenarios have been proposed to account for the the anomalous behavior of the thermodynamic response functions on cooling, each predicting a different behavior for the liquid spinodal, the line of the limit of stability separating the region where liquid water is metastable from the region where the liquid is unstable. (i) According the stabilitylimit conjecture $[10,11]$, the pressure of the spinodal line should decrease on cooling, become negative, and increase again after passing through a minimum. It reenters the positive pressure region of the phase diagram at a very low temperature, thereby giving rise to a line of singularities in the positive pressure region, and consequently the increase in the thermodynamic response functions on cooling in the anomalous region is due to the proximity of this reentrant spinodal. (ii) The critical point hypothesis $[12,13,14,15,16,17]$, proposes a new critical point at the terminus of a first-order phase transition line separating two liquid phases of different density. The anomalous increases of the response functions, compressibility, specific heat, and volume expansivity, is interpreted in terms of this critical point. (iii) The singularity-free hypothesis $[9,18,19]$ proposes that actually there is no divergence close to the anomalous region; the response functions grow on lowering temperature but remain finite, attaining maximum values.

Water properties and anomalies can be strongly influenced by the physical or chemical properties of the medium $[1,3,4,5,20,21]$. The effect not only of applied pressure, but also of negative pressure ("stretching") is remarkable. The study of the behavior of this fluid under nega- 
tive pressures is relevant not only from the academic point of view, but also for realistic systems. For example, negative pressures are observed [22], and seem to play an important role in the mechanism of water transport in plants. Therefore, properties that modify the structure of water, especially if this modification is similar to the effect of stretching (as is the case in some hydrogels [20]), also influence its dynamical behavior.

Dynamic properties, such as the diffusion constant, have been studied in detail for water systems at atmospheric and at high positive pressures, both experimentally $[23,24]$ as well as by computer simulations $[25,26,27,28,29,30,31]$. The increase of pressure increases the presence of defects and of interstitial water molecules in the network [26]. They disrupt the tetrahedral local structure, weakening the hydrogen bonds, and thus increasing the diffusion constant [30, 31]. However, a further increase in the pressure leads to steric effects which works in the direction of lowering the mobility. The interplay of these factors leads to a maximum in the diffusion constant $[30,31]$ at some high density $\rho_{\max }$. Above this density (or corresponding pressure), the diffusion of water is in some sense like that of a normal liquid, controlled by hindrance, with the hydrogen bonds playing a secondary role. However, the behavior at very low $\rho$ is less well understood.

\section{Location of the Spinodal at Positive and Negative Pressures}

Relatively few experimental works $[32,33]$ and simulations $[12,16$, $29,30,35,34]$ have been performed on "stretched" water. In this negative pressure region of the phase diagram the system is metastable, and becomes unstable beyond the spinodal line, so locating the spinodal we can ensure that the simulated state points lie in the metastable and not in the unstable region. Moreover, the shape of the spinodal can test the stability-limit conjecture against the critical point hypothesis and the singularity-free interpretation, so we first discuss the density and pressure of the spinodal, which we denote $\rho_{\mathrm{sp}}(T)$ and $P_{\mathrm{sp}}(T)$, respectively.

Yamada and her coworkers [36] simulated a system of $N=343$ molecules interacting with the TIP5P potential [37]. TIP5P is a fivesite, rigid, non-polarizable water model, not unlike the ST2 model [38]. The TIP5P potential accurately reproduces the density anomaly at 1 atm and exhibits excellent structural properties when compared with experimental data $[37,39]$. The TMD shows the correct pressure dependence, shifting to lower temperatures as pressure is increased. Under ambient conditions, the diffusion constant is close to the experimental 
value, with reasonable temperature and pressure dependence away from ambient conditions [37]. Equilibration runs were performed at constant

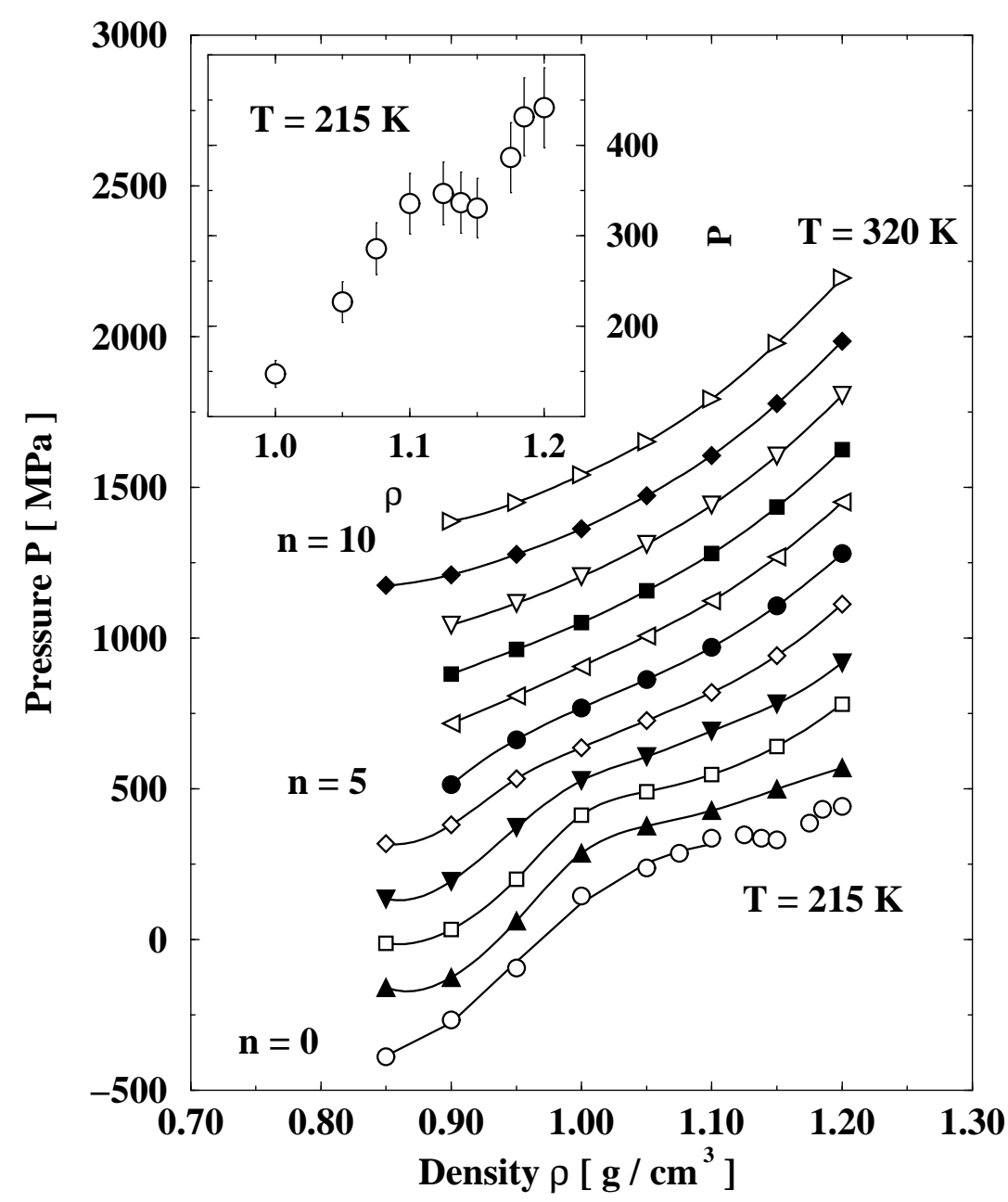

Figure 1. Dependence on density of the pressure at all temperatures investigated ( $T=215,220,230,240,250,260,270,280,290,300,320 \mathrm{~K}$, from bottom to top). Each curve has been shifted by $n \times 150 \mathrm{MPa}$ to avoid overlaps. An inflection appears as T is decreased, transforming into a "flat" coexistence region at $T=215 \mathrm{~K}$, indicating the presence of a liquid-liquid transition. Inset: A detailed view of the $T=215 \mathrm{~K}$ isotherm. Adapted from [36].

$T$. After thermalization at $T=320 \mathrm{~K}$ the thermostat temperature was set to the temperature of interest. The system evolved for a time 
longer than the structural relaxation time $\tau_{\alpha}$, defined as the time at which $F_{s}\left(Q_{0}, \tau_{\alpha}\right)=1 / e$, where $F_{s}\left(Q_{0}, t\right)$ is the self-intermediate scattering function evaluated at $Q_{0}=18 \mathrm{~nm}^{-1}$, the location of the first peak of the static structure factor. In the time $\tau_{\alpha}$, each molecule diffuses on average a distance of the order of the nearest neighbor distance. We use the final configuration of the equilibration run to start a production run of length greater than several $\tau_{\alpha}$ and then analyze the calculated trajectory.

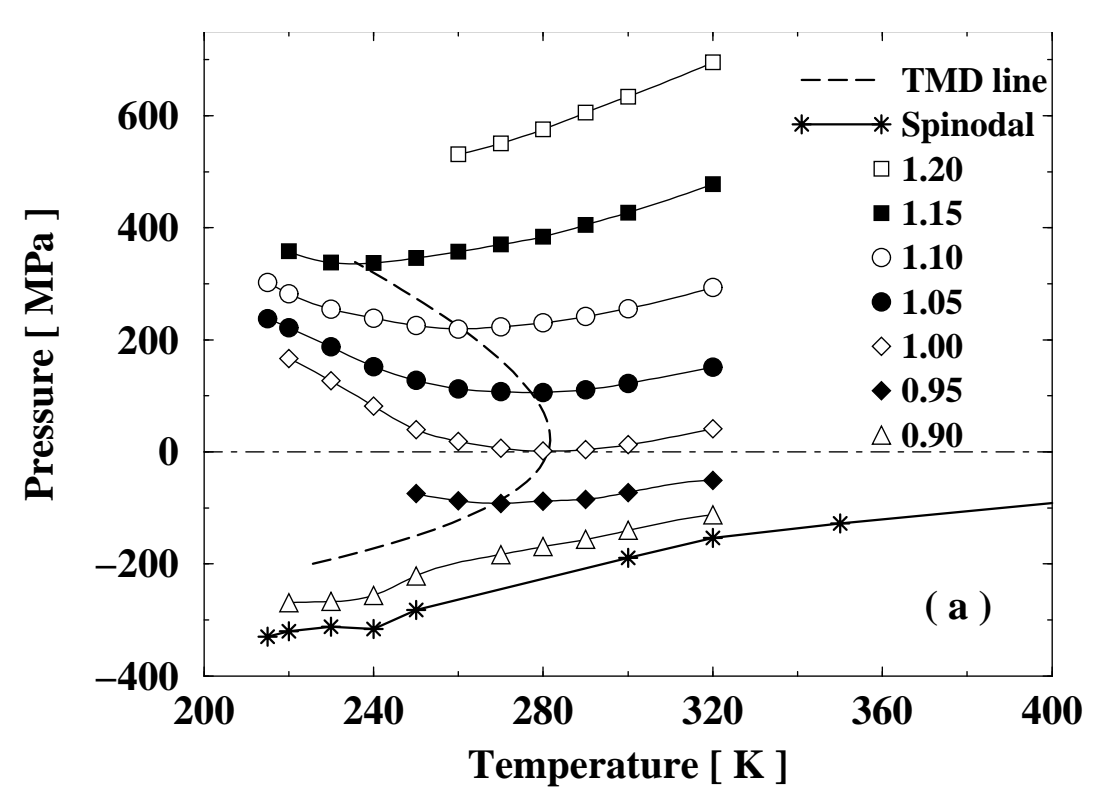

Figure 2. Pressure along seven isochores; the minima correspond to the temperature of maximum density line (dashed line). Note the "nose" of the TMD line at $T=4$ C. Stars denote the liquid spinodal line, which is not reentrant, and terminates at the liquid-gas critical point. Adapted from [36].

Figure 1 shows results for pressure along isotherms. At lower temperatures an inflection develops, which becomes a "flat" isotherm at the lowest temperature, $T=215 \mathrm{~K}$. The presence of a flat region indicates that a phase separation takes place; the critical temperature is $T_{C^{\prime}}=217 \pm 3 \mathrm{~K}$, the critical pressure is $P_{C^{\prime}}=340 \pm 20 \mathrm{MPa}$, and the critical density $\rho_{C^{\prime}}=1.13 \pm 0.04 \mathrm{~g} / \mathrm{cm}^{3}$.

Figure 2(a) plots the pressure along isochores. The curves show minima as a function of temperature; the locus of the minima is the TMD line, since $(\partial P / \partial T)_{V}=\alpha_{P} / K_{T}$. Note that the pressure exhibits a mini- 
mum if the density passes through a maximum $\left(\alpha_{p}=0\right)$. It is clear that, as in the case of ST2 water, TIP5P water has a TMD that changes slope from negative to positive as $\mathrm{P}$ decreases. Notably, the point of crossover between the two behaviors is located at ambient pressure, $T \approx 4 \mathrm{C}$, and $\rho \approx 1 \mathrm{~g} / \mathrm{cm}^{3}$.

Also plotted the spinodal line, obtained by fitting the isotherms (for $T \geq 300 K)$ of Fig. 1 to the form $P(T, \rho)=P_{s}(T)+A\left[\rho-\rho_{s}(T)\right]^{2}$, where $P_{s}(T)$ and $\rho_{s}(T)$ denote the pressure and density of the spinodal line. This functional form is the mean field prediction for $P(\rho)$ close to a spinodal line. For $T \leq 250 K, P_{s}(T)$ is calculated by estimating the location of the minimum of $P(\rho)$. The results in Fig.2 show that the liquid spinodal line is not reentrant and does not intersect the TMD line.

\section{Dynamic Properties}

We next discuss results on the dynamics of stretched water recently obtained by Netz and his collaborators [40]. While there are a large number of intermolecular potential functions used to simulate water, each of which gives slightly different results, the overall thermodynamics picture obtained from these models is generally very similar. Since dynamic properties are particularly sensitive to the potential choice, the extended simple point charge (SPC/E) potential is used since it reproduces both the maximum in diffusivity under pressure as well as the power-law behavior of dynamics properties on cooling. For understanding the properties of water at negative pressure, simulations are particularly important since experiments are very difficult to perform in this region.

The effect of extreme conditions on the flow of the liquid is assessed by calculating the diffusion constant $D$, defined by the asymptotic value of the slope of the mean square displacement versus time. We show $D$ along isotherms in Fig. 3. For $T \leq 260 \mathrm{~K}, D$ has a minimum value at $\rho \approx$ $0.9 \mathrm{~g} / \mathrm{cm}^{3}$, which becomes more pronounced at lower $T$. This behavior can be understood considering the structural changes that occur with decreasing density. At low $T$, the decreased density enhances the local tetrahedral ordering, which leads to a decrease in $D$. Further decreases in density reduces the stability of the tetrahedral structure and causes an increase of $D$.

The location of the minimum is near the ice Ih density $\approx 0.915 \mathrm{~g} / \mathrm{cm}^{3}$, which is the density where the perfect tetrahedral order occurs. The behavior of the minimum of $D, D_{\min }(T)$, complements the known behavior of $D_{\max }(T)$ for the same model [30,31,35], where a maximum 


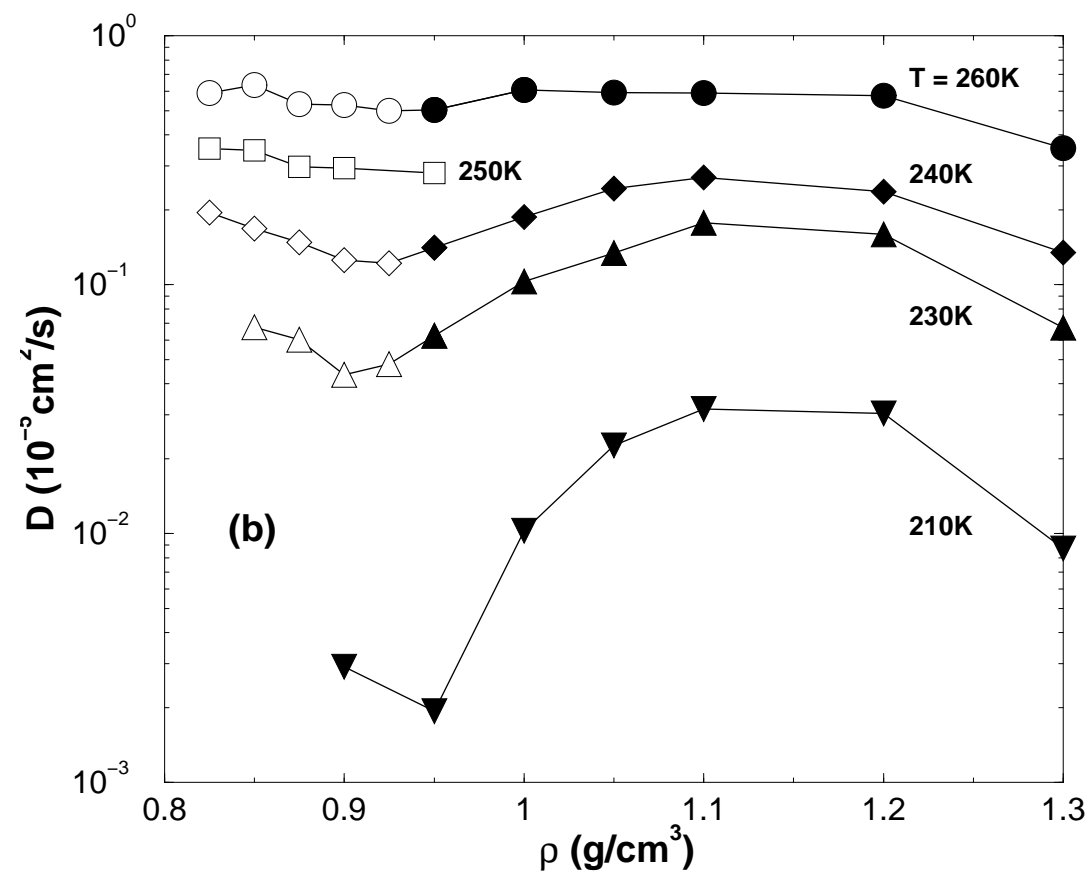

Figure 3. Dependence of the diffusion constant $D$ on $\rho$ along isotherms (for $\rho \leq$ $\left.1.0 \mathrm{~g} / \mathrm{cm}^{3}\right)$. Open symbols are the new simulations we report, and filled symbols are from Ref. [30]. Adapted from [40].

occurs due to breaking hydrogen bonds at high pressure; the density of the $D_{\min }(T)$ increases slightly with increasing $T$, while the density of $D_{\max }(T)$ decreases with increasing $T$ [31]. This is expected, since the range of densities where anomalous behavior occurs expands with decreasing $T$. We show the loci of $D_{\min }(T)$ and $D_{\max }(T)$, along with the spinodal and locus of density maxima in Fig. 4.

Below the spinodal, $D$ also increases, since the mobility of the gas is larger than that of the liquid. However, the simulations clearly show that $D_{\min }$ for the liquid occurs prior to the onset of cavitation, and so the location of $D_{\min }$ we estimate is not affected by phase separation. Recently, Ref. [35] estimated the location of $D_{\min }$ for the same model along several isotherms, and associated $D_{\min }$ with a maximum in orientational order. 


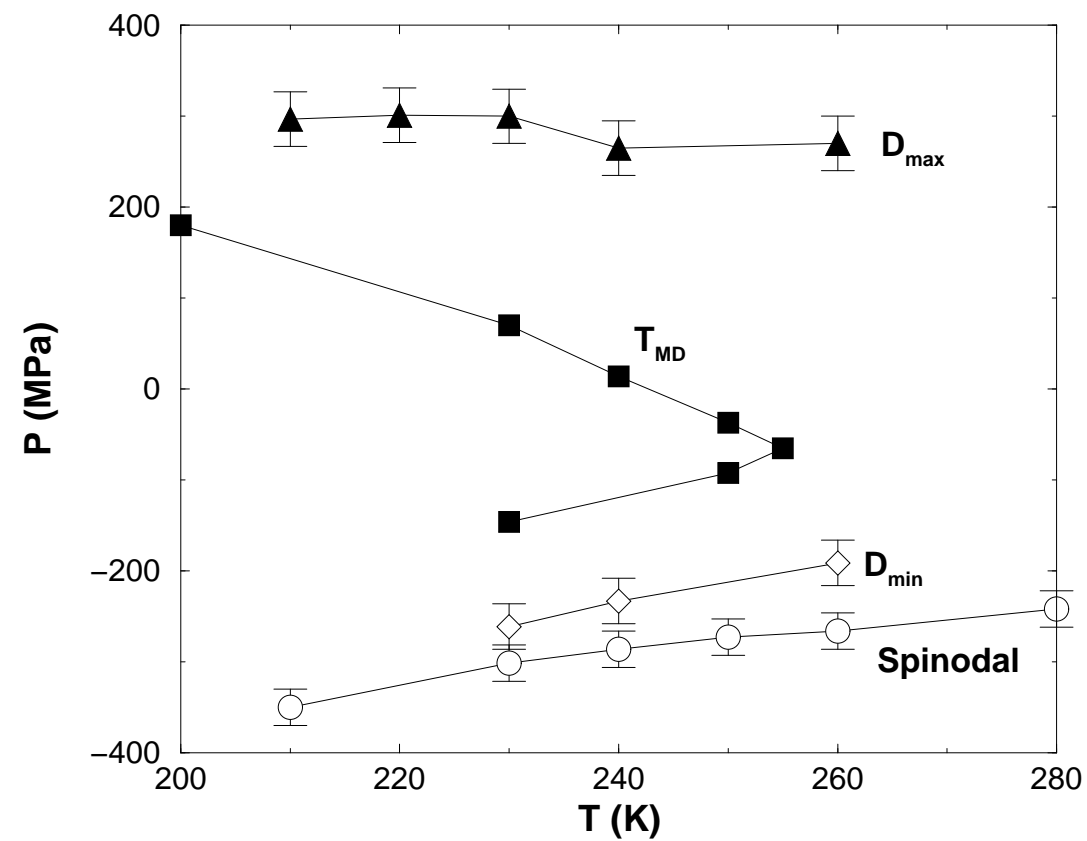

Figure 4. Relation of the loci of maxima and minima of $D$ with $T_{M D}$ and the spinodal. Open symbols are from the present work, and filled symbols are from Ref. [30]. Adapted from [40].

\section{Conclusions}

Water exhibits a very complex structure and its properties and anomalies are strongly influenced by variations of pressure. For high densities $\left(\rho>\rho_{\max }\right)$, water behaves as a normal liquid and the decrease of $D$ with increasing pressure is governed by steric effects. For $\rho_{\min }<\rho<\rho_{\max }$, as the pressure is decreased, the presence of defects and interstitial water decrease, the tetrahedral structure dominates, with stronger hydrogen bonds. This process reaches its maximum at $\rho=\rho_{\min } \approx \rho_{\text {ice. }}$ Further stretching destabilizes the hydrogen bond network, leading to an increase in mobility. The locus of $D_{\text {min }}$ roughly tracks the spinodal, not surprising since the same breakdown of tetrahedral order that gives rise to $D_{\min }$ also facilitates cavitation.

\section{Acknowledgments}

We thank D. R. Baker, S. V. Buldyrev, P. Debenedetti, G. Franzese, W. Kob, E. La Nave, M. Marquez and C. Rebbi for useful discussions, 
and NSF Grant CHE-0096892, the Conselho Nacional de Desenvolvimento Cientifico e Technologico $(\mathrm{CNPq})$, the Fundacao de Amparo a Pesquisa do Rio Grande do Sul (Fapergs) for support. MY thanks NSF Grant GER-9452651 for support as a Graduate Research Trainee at the Boston University Center for Computational Science, FS thanks MURST COFIN 2000 and INFM Iniziativa Calcolo Parallelo, and FWS thanks the National Research Council.

\section{References}

[1] For elementary introductions to recent work on liquid water, the reader may wish to consult P. Ball, Life's Matrix: A Biography of Water (Farrar Straus and Giroux, New York, 2000) or P. G. Debenedetti and H. E. Stanley, "The Novel Physics of Water at Low Temperatures", Physics Today (submitted).

[2] V. Brazhkin. S. V. Buldyrev, V. N. Ryzhov, and H. E. Stanley [eds], New Kinds of Phase Transitions: Transformations in Disordered Substances Proc. NATO Advanced Research Workshop, Volga River (Kluwer, Dordrecht, 2002).

[3] O. Mishima and H. E. Stanley, Nature 396, 329 (1998).

[4] M.-C. Bellissent-Funel, ed., Hydration Processes in Biology: Theoretical and Experimental Approaches (IOS Press, Amsterdam, 1999).

[5] H. E. Stanley, S. V. Buldyrev, N. Giovambattista, E. La Nave, A Scala, F. Sciortino, and F. W. Starr, [Proc. IUPAP Statphys21, Cancun] Physica A 306, 230-242 (2002).

[6] S. V. Buldyrev, G. Franzese, N. Giovambattista, G. Malescio, M. R. SadrLahijany, A. Scala, A. Skibinsky, and H. E. Stanley [Proc. International Conf. on Scattering Studies of Mesoscopic Scale Structure and Dynamics in Soft Matter] Physica A 304, 23-42 (2002).

[7] R. C. Dougherty and L. N. Howard, J. Chem. Phys. 109, 7379 (1998).

[8] A. Geiger, F. H. Stillinger, and A. Rahman, J. Chem. Phys. 70, 4185 (1979).

[9] H. E. Stanley and J. Teixeira, J. Chem. Phys. 73, 3404 (1980).

[10] R. J. Speedy, J. Chem. Phys. 86, 982 (1982); Ibid 86, 3002 (1992).

[11] R. J. Speedy, J. Chem. Phys. 91, 3354 (1987).

[12] P. H. Poole, F. Sciortino, U. Essmann, and H. E. Stanley, Nature 360, 324 (1992); Phys. Rev. E 48, 3799 (1993); F. Sciortino, P. H. Poole, U. Essmann, and H. E. Stanley, Ibid. 55, 727 (1997); S. Harrington, R. Zhang, P. H. Poole, F. Sciortino, and H. E. Stanley, Phys. Rev. Lett. 78, 2409 (1997).

[13] O. Mishima, J. Chem. Phys. 100, 5910 (1994).

[14] P. H. Poole, F. Sciortino, T. Grande, H. E. Stanley and C. A. Angell, Phys. Rev. Lett. 73, 1632 (1994); C. F. Tejero and M. Baus, Phys. Rev. E 57, 4821 (1998); T. M. Truskett, P. G. Debenedetti, S. Sastry, and S. Torquato, J. Chem. Phys. 1112647 (1999).

[15] M.-C. Bellissent-Funel, Europhys. Lett. 42, 161 (1998); O. Mishima and H. E. Stanley, Nature 392, 192 (1998).

[16] H. Tanaka, J. Chem. Phys. 105, 5099 (1996). 
[17] A. Scala, F. W. Starr, E. La Nave, H. E. Stanley and F. Sciortino, Phys. Rev. E 62, 8016 (2000).

[18] S. Sastry, P. G. Debenedetti, F. Sciortino, and H. E. Stanley, Phys. Rev. E 53, 6144 (1996).

[19] L. P. N. Rebelo, P. G. Debenedetti, and S. Sastry, J. Chem. Phys. 109, 626 (1998).

[20] P. A. Netz and Th. Dorfmüller, J. Phys. Chem. B 102, 4875 (1998).

[21] K. Koga, X. C. Zeng, and H. Tanaka, Chem. Phys. Lett. 285, 278 (1998).

[22] W. T. Pockman, J. S. Sperry, and J. W. O'Leary, Nature 378, 715 (1995).

[23] J. Jonas, T. DeFries, and D. J. Wilbur, J. Chem. Phys. 65, 582 (1976).

[24] F. X. Prielmeier, E. W. Lang, R. J. Speedy, and H.-D. Lüdemann, Phys. Rev. Lett. 59, 1128 (1987); Ber. Bunsenges. Phys. Chem. 92, 1111 (1988).

[25] M. Rami Reddy and M. Berkovitz, J. Chem. Phys. 87, 6682 (1987).

[26] F. Sciortino, A. Geiger, and H. E. Stanley, Nature 354, 218 (1991); Ibid., J. Chem. Phys. 96, 3857 (1992).

[27] N. Giovambattista, F. W. Starr, F. Sciortino, S. V. Buldyrev, and H. E. Stanley, Phys. Rev. E 65, 041502-1 - 041502-6 (2002) cond-mat/0201028.

[28] E. La Nave, A. Scala, F. W. Starr, H. E. Stanley and F. Sciortino, Phys. Rev. E 64, 036102-1 - 036102-10 (2001); E. La Nave, H. E. Stanley and F. Sciortino, Phys. Rev. Letters 88, 035501-1 to 035501-4 (2002) cond-mat/0108546.

[29] P. Gallo, F. Sciortino, P. Tartaglia, and S.-H. Chen, Phys. Rev. Lett. 76, 2730 (1996).

[30] F. W. Starr, F. Sciortino, and H. E. Stanley, Phys. Rev. E 60, 6757 (1999); F. W. Starr, S. T. Harrington, F. Sciortino, and H. E. Stanley, Phys. Rev. Lett., 82, 3629, (1999).

[31] A. Scala, F. W. Starr, E. La Nave, F. Sciortino and H. E. Stanley, Nature 406, 166 (2000).

[32] S. J. Henderson and R. J. Speedy, J. Phys. E: Scientific Instrumentation 13, 778 (1980).

[33] J. L. Green, D. J. Durben, G. H. Wolf, and C. A. Angell, Science 249, R649 (1990).

[34] I. I. Vaisman, L. Perera, and M. L. Berkovitz, J. Chem. Phys. 98, 9859 (1993).

[35] J. R. Errington and P. G. Debenedetti, Nature 409, 318 (2001).

[36] M. Yamada, S. Mossa, H. E. Stanley, F. Sciortino, Phys. Rev. Letters 88, 195701 (2002); cond-mat/0202094

[37] M. W. Mahoney and W. L. Jorgensen, J. Chem. Phys. 112, 8910 (2000); Ibid. 114, 363 (2001).

[38] F. H. Stillinger and A. Rahman, J. Chem. Phys. 60, 1545 (1974).

[39] J. M. Sorenson, G. Hura, R. M. Glaeser, and T. Head-Gordon, J. Chem. Phys. 113, 9149 (2000).

[40] P. A. Netz, F. W. Starr, H. E. Stanley, and M. C. Barbosa, J. Chem. Phys. 115, 344-348 (2001); cond-mat/0102196; P. A. Netz, F. W. Starr, H. E. Stanley, and M. C. Barbosa, cond-mat/0201130; P. A. Netz, F. Starr, M. C. Barbosa, H. E. Stanley, cond-mat/0201138. 